\title{
Configuration Optimization Method of Reconfigurable Manipulator based on Dijkstra Algorithm
}

\author{
Xinyu Liu ${ }^{1, \mathrm{a}}$, Bo Dong ${ }^{1, \mathrm{~b}}$, Keping Liu ${ }^{1, \mathrm{c}}$ and Yuanchun $\mathrm{Li}^{1, \mathrm{~d}^{*}}$ \\ ${ }^{1}$ Department of Control Engineering, Changchun University of Technology, Changchun 130012, \\ China
}

aliuxy07@hotmail.com, dongbo@ccut.edu.cn, ${ }^{9}$ liukeping@ccut.edu.cn ${ }^{d}$ liyc@ccut.edu.cn

\begin{abstract}
Keywords: Reconfigurable manipulator, Configuration optimization, Genetic algorithm, Dijkstra algorithm, Shortest path of directed graph.

Abstract. In this paper, a novel configuration optimization method is proposed for reconfigurable manipulator. The optimize configuration of reconfigurable manipulator is proposed by using the genetic algorithm and the Dijkstra algorithm. Considering both minimum task execution time and minimum energy consumption, the evaluation criteria of genetic algorithm is formed inverse kinematic solution of reconfigurable manipulator. Based on general expression of joint transform matrix of reconfigurable manipulator, inverse kinematics problem is transformed into the problem of shortest path problem in directed graph. The virtual module is introduced to optimize the degree of freedom (DOF) of the manipulator Configuration. Finally, the results of the optimization analysis are verified by the simulation results.
\end{abstract}

\section{Introduction}

Traditional manipulator is developed according to the specific application task. It meets the needs of the application tasks at that time, but with the development of science and technology, the manipulator is applied more and more widely, the traditional manipulator of the single configuration has been unable to meet the demand for changes in the market. In order to solve the relationship between the performance and structure of the manipulator, a new kind of manipulator, reconfigurable modular manipulator, reflects better adaptability to different working environment and work scope by changing the configuration. How to find out the structure which coverage more extensive, the less system module, reconfigurable operation is more simple has been an important subject of research on reconfigurable manipulator. According to different task requirements, the reconfigurable manipulator can be free to increase or decrease module, and transform the connection method. Many existing methods for configuration design, but only limited to the completion of a specific task without considering the generality of configuration design. Therefore, it is necessary to find a way to construct the structure based on task reachability and general purpose.

Genetic algorithm has been widely used in the optimization problem of reconfigurable manipulator. Because of its own characteristics, the search speed of genetic algorithm is relatively slow, and it needs more time to get more accurate solution. In order to avoid these disadvantages, the genetic algorithm is used by combining with other intelligent algorithms. In the process of manipulator configuration optimization with genetic algorithm, [1] introduced the concept of temporary joint module. [2] proposed a new configuration called Configuration Connection Matrix (CCM), and used a method combined genetic algorithm with simulated annealing algorithm for reconfigurable manipulator configuration optimization. In this paper, the change of the joint angle of the reconfigurable manipulator is used as the evaluation criterion of the genetic algorithm. The inverse kinematics solution of the manipulator includes a closed solution and a numerical solution. The closed solution is high accuracy, fast speed and easy to determine all possible solutions, but it is more dependent on the specific geometrical features of the manipulator specific mechanism, suitable for solving the inverse kinematics of manipulator with a simple structure model. The numerical solution include based on Jacobi matrix solution, neural network solution, genetic algorithm and so on. By the structure plane matching method, [3] proposed a solution for solving the inverse kinematics of reconfigurable 
manipulator. [4] proposed a hybrid electromagnetic mechanism based on electromagnetic mechanism and pattern search. The method of REM algorithm and PS search method solved the global exploration and local exploitation ability of the algorithm. [5] proposed a configuration transformation method by changing the parameters of D-H method. In order to reconstruct real-time and adaptive, a strategy for control system and mechanical system was presented in [6]. These methods are the inverse kinematics solution of a simple manipulator, which is not suitable for the combination of genetic algorithm. With the development of the mathematical programming method and computer, it is possible to analyze the structure of complex problems naturally with a new optimization strategy. By combining the standard method with the mathematical programming method, [7] shown that the results obtained by the method of the dual column are similar to those obtained by the standard method. So it is feasible to combine genetic algorithm with Dijkstra algorithm to solve the problem of configuration optimization. It does not only solve the problem of slow speed of the genetic algorithm, but also guarantees the accuracy of the solution.

In this paper, the inverse kinematics of the manipulator is used as the evaluation criterion, and optimizing reconfigurable manipulator configuration with the genetic algorithm. The inverse kinematics of the manipulator is transformed to the problem of the shortest path of directed graph. To combine genetic algorithm with Dijkstra algorithm, it is not only reduces the computational difficulty of mathematical methods in the multi-variable problem, but also avoids the problem of the genetic algorithm to search the slow and easy to fall into local optimum. The concept of virtual module is introduced to optimize the DOF of reconfigurable manipulator. Finally, the optimization method is verified by simulation.

\section{Kinematic Model of Manipulator}

The design of the manipulator modular joint needs to consider the structure and size, also to have a good generality and interchange. In Fig. 1, take 6-DOF reconfigurable manipulator of xPartner Evolutionary Force ${ }^{\mathrm{TM}}$ EV-M robot Series as an example. The module can be divided into joint module and link module. The joint module connected with different link module forms the rotary joint and swinging joint.

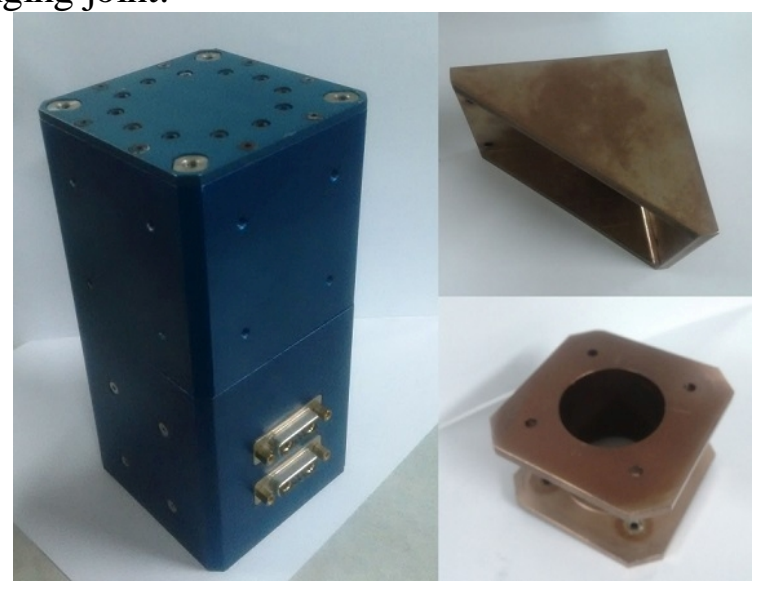

Fig. 1 Joint Module and Link Module

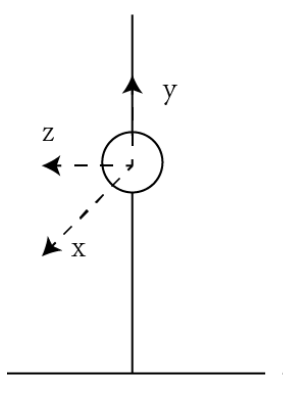

(a)Swinging Joint

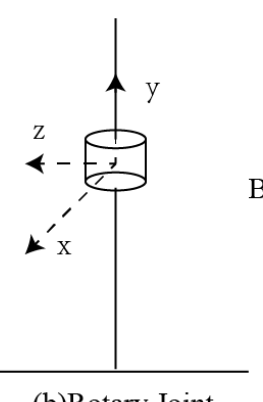

(b)Rotary Joint

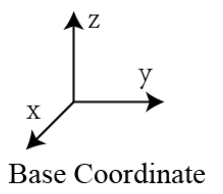

Fig. 2 Joint Module Coordinate

Based on modular thought of reconfigurable manipulator, a kinematic model for each module is established by using the modified D-H parameter method. The joint module has only one variable, and the connection module has no variable. Connecting mechanism connect between modules. In order to facilitate the expression, the unified expression of the reconfigurable manipulator module is established. 


$$
T=\left[\begin{array}{cccc}
\cos \theta & -\sin \theta \sin \beta & -\sin \theta & -h \sin \theta \sin \beta \\
0 & \cos \beta & -\sin \beta & h \cos \beta+l \\
\sin \theta & \cos \theta \sin \beta & \cos \theta \cos \beta & h \cos \theta \sin \beta \\
0 & 0 & 0 & 1
\end{array}\right]
$$

Where, $\theta$-The rotation angle of rotary joint, it is zero for other modules;

$\beta$-The rotation angle of swinging joint, it is zero for other modules;

$h$-The connecting length of swinging joint, it is zero for other modules;

$l$-The connecting length of rotary joint, it is zero for other modules.

As shown in Fig. 2, the coordinate for different joints established. In the initial state, the coordinate only needs to rotate $90^{\circ}$ anticlockwise around the $\mathrm{X}$ axis. The unified expression of the reconfigurable manipulator module can be simplify to the following form, it reduces the computational difficulty

$$
T=\left[\begin{array}{cccc}
\cos \theta_{i} & 0 & -\sin \theta_{i} & 0 \\
0 & \cos \beta & -\sin \beta_{i} & h \cos \beta_{i}+l \\
\sin \theta_{i} & \cos \theta_{i} \sin \beta_{i} & \cos \theta_{i} \cos \beta_{i} & h \cos \theta_{i} \sin \beta_{i} \\
0 & 0 & 0 & 1
\end{array}\right](i=1 \mathrm{~L} n)
$$

By the unified expression of the basic module, the model of the mechanical arm is set up in the form of the model of the mechanical arm:

$$
T=\prod_{i=1}^{n}{ }^{i} T_{i-1}=\left[\begin{array}{cc}
R & P \\
0 & 1
\end{array}\right]
$$

\section{Design of Configuration Optimization Method}

\section{Implementation of configuration optimization based on the genetic algorithm}

Genetic algorithm uses the probability of optimization method, can automatically obtain and guide the optimization of search space, adaptively adjusts the search direction, does not need to determine the rules. It is widely applied to the domain of combinational evolutionary problem seeking, self-adapt controlling and machine learning. In this paper, genetic algorithm is used to optimize the configuration of reconfigurable manipulator, a unified evaluation index is needed for the reconfigurable manipulator with different configuration. Select the following two indicators: the amount of accessibility and the angle of each joint.

Select the accessibility, reconstruction of the difficulty, the variation of joint angles as the evaluation index. The advantage of this method is that the reconstruction of reconfigurable manipulator can be more easily and quickly. The change of joint angle is used as the evaluation criterion, and the task execution time and energy consumption are considered comprehensively. The fitness function is selected as follow:

$$
\text { Fit } V=\frac{1}{|q|+k N_{L}}
$$

Where, $q$ is the angle of each joint, $|q|$ is the 1 paradigm of the angle of each joint, $k$ is the weight between the DOF and the angle of each joints, $N_{L}$ is the DOF of final configuration.

The power law is introduced in the design of the genetic algorithm selection operator, in the t-th generation, the probability of being chosen as an individual is

$$
P\left\{T_{s}\left(\stackrel{r}{X^{t}}\right)=X_{i}^{t}\right\}=\frac{f\left(X_{i}^{t}\right)}{\sum_{j=1}^{n} f\left(X_{i}^{t}\right)}
$$


Cross method selects one point crossover, the crossover operator is

$$
P\left\{T_{c}\left(X_{1}, X_{2}\right)=Y\right\}=\left\{\begin{array}{cc}
\frac{k p}{l}, & Y \neq X_{1} \\
\left(1-p_{c}\right)+\frac{k p}{l}, & Y=X_{1}
\end{array}\right.
$$

For any two individuals $X, Y \in S$, the mutation operator is chosen as

$$
P\left\{T_{c}\left(X_{1}, X_{2}\right)=Y\right\}=p_{m}^{d(X, Y)}\left(1-p_{c}\right)^{1-d(X, Y)}
$$

\section{Solution of Inverse Kinematics based on the Dijkstra algorithm}

The reconfigurable manipulator is usually used in series configurations, a variety of configurations can be accomplished for the same task as shown in Fig. 3. The reconfigurable manipulator configuration can be regarded as a connected directed graph. Taking the center of the base of the reconfigurable manipulator as the start point, the destination is the end of the reconfigurable manipulator, joint angle is the weight of the directed edge. Then the problem of the inverse kinematics of the manipulator is equivalent to the problem of the shortest path of the directed graph.

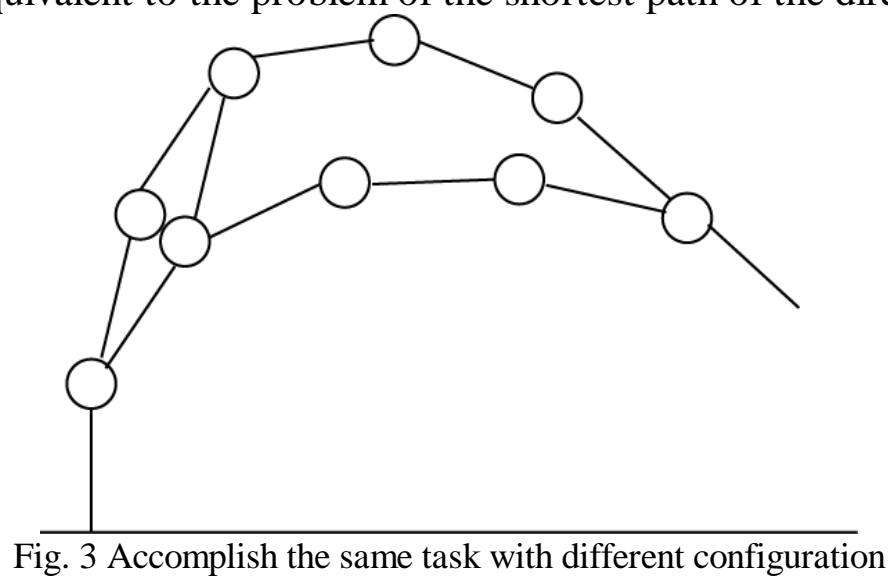

Obviously, there is a linear relationship between the given task matrix and the positive motion model. Therefore, the inverse kinematics of reconfigurable manipulator is obtained by using the Dijkstra algorithm, and the inverse solution is used as the evaluation criterion of genetic algorithm. Set the target function according to the evaluation criteria. Finally, the optimal configuration is obtained.

The optimization objective is minimum execution time and minimum energy consumption as follow.

$$
\min \quad J=\|w \theta\|
$$

Where, $w$ is a diagonal matrix, $w_{i}$ is the value of angle of the $\mathrm{i}$-th joint, $w_{i}$ is the weight value is larger, and the cost of rotation is relatively larger. D. E. Whitney proposed the concept of weighted kinetic energy:

$$
\frac{1}{2} \theta^{T} w^{T} w \theta
$$

It is unified with the objective function. While the objective function is minimum, the system's kinetic energy is also minimum.

$$
r=f(\theta)
$$

Where, $r=\left\{p_{1}, p_{2}, p_{3}\right\}, \theta=\left\{\theta_{1}, \theta_{2} \mathrm{~L} \theta_{i}\right\}$. Differentiate on both sides of the equation.

$$
\begin{gathered}
r^{\prime}=J(\theta) \theta^{\prime} \\
\theta^{\prime}=J^{-1}(\theta) r^{\prime}
\end{gathered}
$$


Where, $J(\theta)$ is Jacobi matrix. When the reconfigurable manipulator is redundant, $J(\theta)$ is not square matrix , $J^{-1}(\theta)$ does not exist. It needs to find $J(\theta)$ pseudo inverse. For the n-DOF manipulator, there is

$$
\theta^{\prime}=w^{-1}\left[j w^{-1}\right]^{-1} r^{\prime}+w^{-1}\left(I-\left[j w^{-1}\right]^{-1} j w^{-1}\right) V
$$

Where , $I$ is a Unit matrix, $V$ is an arbitrary n-dimensional vector, $[\bullet]^{-1}$ is pseudo inverse operation. $w$ is weight diagonal matrix. Due to the existence of redundant degrees of freedom, there are many Kinematic inverse solutions which is the value of the directed graph edge of the reconfigurable manipulator configuration problem. According to the Dijkstra algorithm, the shortest distance between the vertices of directed graph is

$$
\operatorname{dist}[j]=\min \{\operatorname{dist}[j], \operatorname{dist}[i]+\operatorname{matrix}[i][j]\}
$$

By the Eq. 13 and Eq. 14, the minimized solution of the objective function is

$$
\left\{\begin{array}{l}
\theta^{\prime}=w^{-1}\left[j w^{-1}\right]^{-1} r^{\prime}+w^{-1}\left(I-\left[j w^{-1}\right]^{-1} j w^{-1}\right) V \\
\theta_{i}=\min \left\{\theta_{i}, \theta_{i-1}+\text { martrix }\left[\theta_{i-1}\right]\left[\theta_{i}\right]\right\}
\end{array}\right.
$$

In summary, manipulator inverse kinematics problem model is established as follow.

$$
\begin{array}{ll}
\min \quad J & =\|w \theta\| \\
\text { s.t. } & \\
\theta^{\prime} & =w^{-1}\left[j w^{-1}\right]^{-1} r^{\prime}+w^{-1}\left(I-\left[j w^{-1}\right]^{-1} j w^{-1}\right) V \\
\theta_{i} & =\min \left\{\theta_{i}, \theta_{i-1}+\text { martrix }\left[\theta_{i-1}\right]\left[\theta_{i}\right]\right\} \\
T & =\prod_{i=1}^{6} T\left(\theta_{i}\right)=\left[\begin{array}{ll}
R & P \\
0 & 1
\end{array}\right]
\end{array}
$$

Combined Genetic algorithm with Dijkstra algorithm, the optimization of the mechanical structure is carried out in two aspects: the minimum time and the minimum energy. Program structure is as shown in Fig. 4.

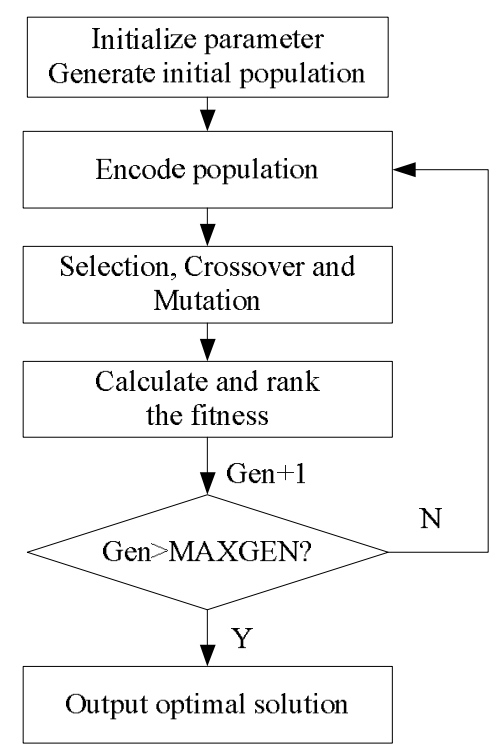

Fig. 4 Program flow chart

\section{Simulation}

The 20 configurations of the reconfigurable manipulator are randomly generated as the initial population of the genetic algorithm, which is formed with a rotary transmitting module, a swinging 
module and a virtual module. The matrix order of each individual in the population is $1 \times 6$. The different joint types are encoded as follows:

Table 1 Module Encoding Mode

\begin{tabular}{cccc}
\hline Module Type & Swinging Joint & Rotaty Joint & Virtual Joint \\
\hline Binary Code & 10 & 01 & 00 \\
\hline
\end{tabular}

Solving the optimal configuration of reconfigurable manipulator can be achieved as follow pose.

$$
T=\left[\begin{array}{cccc}
0.1549 & -0.6656 & 0.7300 & 23.9791 \\
0.3166 & 0.6665 & 0.6749 & -41.6116 \\
-0.9358 & -0.3357 & 0.1075 & 11.2253 \\
0 & 0 & 0 & 1.0000
\end{array}\right]
$$

Optimization design parameters: Population quantity $N_{1}=20$; Genetic algebra MAXGEN=50 ; Crossover probability $p_{c}=0.7$; Mutation probability $p_{m}=0.07$; Joint angle limit: $-2.79 \mathrm{rad} \leq \theta \leq 2.79 \mathrm{rad}$. When the configuration is not satisfied, the fitness function is assigned a maximum value. Simulation results are as Fig.5:

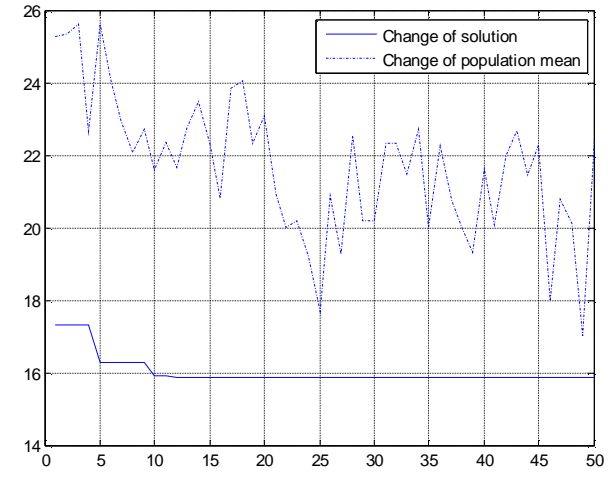

Fig. 5 Matlab simulation results

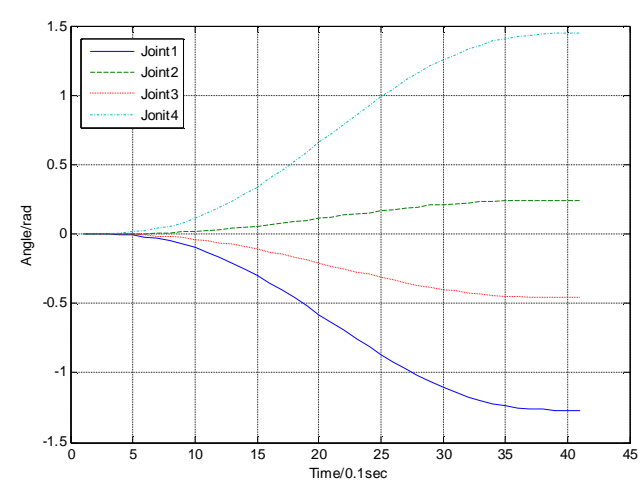

Fig. 6 Time varying curve of joint angle

As shown in Fig. 5, optimal results are obtained in the twelfth generation, is 15.8749. The structure is formed by two swinging joints and two rotary joints. Compared to the previous 6-DOF reconfigurable manipulator, it reduces 2 DOF. Configuration description is

$$
\text { asm }=\left[\begin{array}{cc}
10 & -1.2715 \\
01 & 0.2451 \\
10 & -0.4588 \\
01 & 1.4470
\end{array}\right]
$$
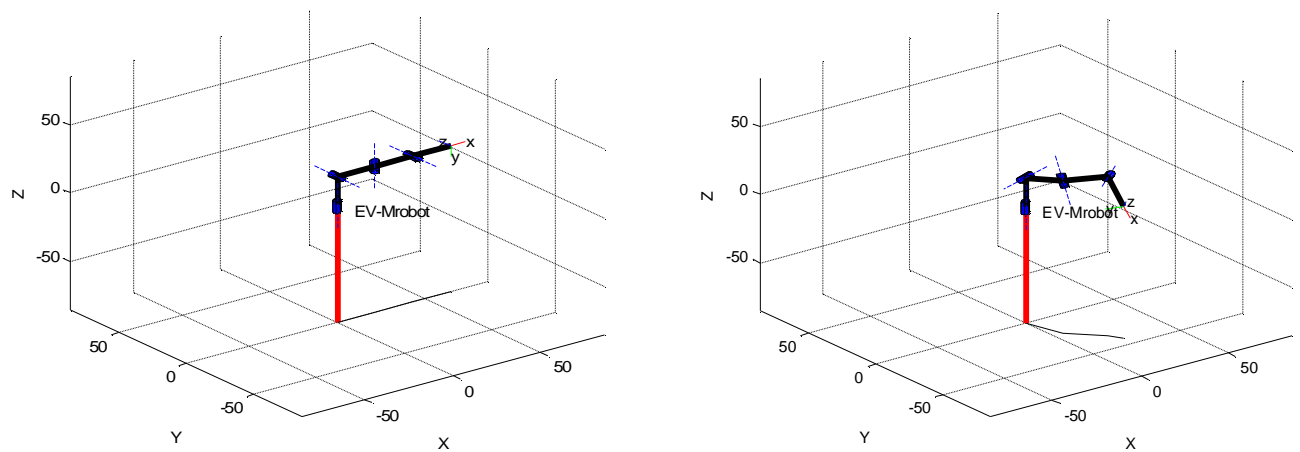

Fig. 7 The initial state and the final state

The maximum angular velocity of joint modular is $30 \% \mathrm{sec}$, Time varying curve of joint angle is shown in Fig. 6.The initial state and the final state of the mechanical arm are as shown in Fig. 7. 


\section{Conclusion}

In this paper, a novel method based on the graph theory in the operations research is proposed. By using generality of solving the inverse kinematics problem of reconfigurable manipulator with different configuration by graph theory method, combined genetic algorithm with Dijkstra algorithm to optimize the structure of reconfigurable manipulator. During the optimization process, consider the two aspects of minimum execution time and minimum energy. The optimization of the DOF reduces difficulty of the reconstruction process by the introduction of virtual modular. By using this method, the optimal assembly mode and joint angle of the target task can be solved simultaneously. Finally, the optimization of the $3 \mathrm{D}$ simulation is verified.

\section{Acknowledgements}

This work is financially supported by: National Natural Science Foundation of China (Grant No. 61374051), State Key Laboratory of Management and Control for Complex Systems (Grant No. 20150102) and The Scientific Technological Development Plan Project in Jilin Province of China (Grant No. 20150520112JH).

\section{References}

[1] Y. J. Wang, Reconfigurable modular robot configuration design theory and kinematics research, North China Electric Power University (Beijing), 2011

[2] B. Dong, Y. CH. Li, Multi-Objective-Based Configuration Generation and Optimization for Reconfigurable Modular Robot. International conference on Information Science and Technology, Nanjing Jiangsu China, March 26-28,2011

[3] Y. H. Wei,J. Zhao, A method for solving the kinematics of reconfigurable robot, Journal of Harbin Institute of Technology, 2010(1) 133-137

[4] Z. W. Ren, Wang Zhenhua, Li Juan, Sun Yaning. Inverse kinematics solution of manipulator based on hybrid electromagnetic mechanism, Journal of Mechanical Engineering, 2012(17)

[5] F. Aghili, K. Parsa. A reconfigurable robot with lockable cylindrical joints. IEEE Transactions on robotics, 2009, 25(4) 785-797

[6] M. H. Wang, S. G. Ma, B. Li, Y. C. Wang, X. Y. He, The design and implementation of reconfigurable planetary robot control system, Robot, 2005,27(3) 273-277

[7] Fleury C. Reconcilation of mathematical programming and optimality criteria method. In Morres A J, ed. Foundation of Structural optimization. New York: Jone Wiley \& Sons, 1982

[8] C. L. Jing, A. H. Han, Application of mathematical principle in Robotics. Innovative Technology, 2013(11) 31-32

[9] Y. S. Kung, M. K. Wu. Design of inverse kine matics IP for a six-axis articulated manipulator. Automatic Control Conference (CACS), 2013 CACS International. 2-4 Dec. 2013

[10] J. Y. He, Operational Research Foundation [M]. Beijing: Tsinghua University press, 2000.17-2 